\title{
Protracted Adjuvant Temozolomide in Glioblastoma Multiforme
}

\author{
Ahmed A. Refae', Ahmed Ezzat', Dina Ahmed Salem', Mervat Mahrous ${ }^{2}$ \\ ${ }^{1}$ Clinical Oncology Department, Ain Shams University, Cairo, Egypt \\ ${ }^{2}$ Clinical Oncology Department, Al Minia University, Al Minia, Egypt \\ Email: ahmedezzat 2000@yahoo.com, Dinasalem70@yahoo.com, mervat mahrous@yahoo.com
}

Received 12 July 2015; accepted 11 August 2015; published 14 August 2015

Copyright (C) 2015 by authors and Scientific Research Publishing Inc.

This work is licensed under the Creative Commons Attribution International License (CC BY). http://creativecommons.org/licenses/by/4.0/

(c) (i) Open Access

\begin{abstract}
Purpose: Radiotherapy with concurrent temozolomide (TMZ), followed by 6 cycles of adjuvant TMZ, is the standard of care for newly diagnosed Glioblastoma Mulltiforme (GBM). However tumor progression is the role with median survival of almost 14 months. With lack of effective second line chemotherapy, many physicians and some guidelines advocate prolonged use of adjuvant TMZ more than 6 months. We conduct this study to test the efficacy of protracted adjuvant conventional dose TMZ over the standard 6 doses of adjuvant TMZ. Material and Methods: This phase II trial enrolled patients newly diagnosed as GBM, older than age 18 years, with a Karnofsky performance score (KPS) of $\geq 60$, Neurological Performance Scale (NPS) of $\leq 3$. Patients were randomly assigned to the standard concurrent chemoradiotherapy (CCRT) followed by 6 cycles of adjuvant TMZ or the same treatment with more than 6 cycles of adjuvant chemotherapy extended as long as the patient in good performance, with no unacceptable toxicity, no signs of disease progression. The primary end point was OS. Results: A total of 59 patients were recruited in the study and were randomized in two arms. 29 patients joined arm 1 aiming at receiving CCRT followed by adjuvant 6 cycles TMZ ( 6 cycles arm) and 30 joined arm 2 aiming at receiving the same treatment with more than 6 cycles of TMZ ( $>6$ cycles). 16 patients managed to complete the adjuvant 6 cycles in arm 1 . 19 patients in arm 2, completed the 6 cycles with additive more doses with a median of 11 cycles (range: 8 - 23 cycles). Median PFS was 12.1 months for (6 cycles) arm, and 18.8 months for ( $>6$ cycles) arm, HR 0.88 (95\% CI: 1.185 - 4.901) (P 0.015); the overall survival for (6 cycles) arm was 18.1 months, versus 24.1 months, HR 0.70 (95\% CI: 1.007 - 4.037) (P 0.048). No significant added toxicity was notice and the 4 weekly TMZ was well tolerated. Conclusion: This study concluded that protracted adjuvant TMZ after concurrent chemoradiotherapy could be a feasible strategy for GBM. This strategy warrants a large phase III randomized trial.
\end{abstract}

\section{Keywords}

Glioblastoma Multiforme, Timozolomide, Protracted Course, Efficacy 


\section{Introduction}

Gliomas are the most common form of malignant primary brain tumors in adults, with an annual incidence of approximately four to five per 100,000 people [1]. It has a poor prognosis despite surgery, radiotherapy (RT) and chemotherapy [2].

Stupp and colleagues (2005) published the result of a phase III study conducted over 573 patients from 85 centers by the European Organization for Research and Treatment of Cancer and the National Cancer Institute of Canada (EORTC/NCIC) comparing between maximum safe resection or biopsy followed by radiotherapy versus adding concurrent temozolomide (TMZ) followed by six cycles of adjuvant TMZ. The median survival was 14.6 months with radiotherapy plus temozolomide and 12.1 months with radiotherapy alone. The two-year survival rate was $26.5 \%$ with radiotherapy plus temozolomide and $10.4 \%$ with radiotherapy alone [3].

The updated 5-years survival result was $9.8 \%$ in the RT with TMZ compared to $1.9 \%$ in RT alone group [4].

These randomized prospective data were the first to demonstrate a significant and meaningful survival benefit when a chemotherapeutic agent was given in combination with RT in this disease hence it constituted the current standard of care for the adjuvant treatment of GBM.

Even though with such improvement in survival with such practice, recurrence is the rule and the majority die within the first 2 years.

In view of paucity of effective second line, and aiming for delaying recurrence and improving survival many oncologists at real practice prescribe more than the standard 6 cycles. This is encouraged by the overall tolerable side effects of the conventional dose schedule of TMZ [5].

Hau and colleagues surveyed neuro-oncologists regarding patients treated with temozolomide for at least 12 cycles. Patients receiving first-line temozolomide for a median of 13 cycles had a median progression-free survival (PFS) of 14 months. A small percentage of patients experienced grade III to IV toxicity, and concluded that these results suggested that long-term treatment with temozolomide was feasible and well tolerated [6]. Seiz and colleagues published a study over 114 patients in a single institution who received the standard CCRT followed by adjuvant TMZ for stable and responder patients. Cycle's number ranged from 1 - 57 cycles. Median OS in all patients was 15 months (95\% CI: 13 - 18 months). TTP and OS directly correlate with the amount of chemotherapy cycles (each $\mathrm{P}<0.0001)$ [7].

Malkoun and colleagues also concluded the feasibility and safety of prolonged adjuvant TMZ in a retrospective study [8]. While Khasraw et al. reported three patients who tolerated TMZ for 5, 7 and 8 years [9]. Moreover some guidelines suggest more cycles or maintenance strategy for patients with a partial response or with continuing radiological improvement at the end of the sixth cycle [10].

So aiming to maximize the benefit of temozolomide (TMZ), which is the backbone in the treatment of brain tumors, we conduct this study to assess the efficacy of protracted adjuvant temozolomide as long as patients do not show disease progression, unacceptable toxicity, and in reasonable general and neurological performance.

A prospective phase II randomized trial in which glioma patients were divided into two groups: group one received post surgery CCRT followed by 6 cycles of adjuvant TMZ (EORTC/NCIC protocol) and the second group received the same treatment with more than 6 cycles of chemotherapy or till disease progression.

Primary endpoint is OS of patients who receive more than 6 cycles of adjuvant versus the standard adjuvant 6 cycles. Secondary endpoint is PFS of the same groups, PFS and OS based on intension-to-treat (ITT) analysis to the two randomized groups.

\section{Material and Method}

Patient accrual started on October 2008 till August 2012. Inclusion criteria included: adult patient, histological proven diagnosis of WHO grade IV astrocytoma, karnofsky performance status of 60 or above, and medical research council (MRC) Neurological Performance Scale (NPS) of 3 or better.

The patients should undergo surgical intervention aiming at maximal safe resection of the gross disease and if not feasible, patient showed have at least biopsy for establishing the diagnosis.

The patient's treatment followed the (EORTIC/NCIC) protocol [3]. They received CCRT for 6 weeks. Radiotherapy aims a total dose of $60 \mathrm{~Gy}$, conventional fractionation, through three-dimensional conformal radiotherapy (3-DCRT) or intensity modulated radiotherapy (IMRT) techniques. Clinical target volume included the gross tumor volume with a $2-3 \mathrm{~cm}$ margin. Smaller margin might be appropriate to avoid sensitive structure in 
presence of anatomical natural barriers that can impede the contiguous spread of tumor cells [11]. It may start 4 - 6 weeks after surgery after removal of the staples or sutures.

Concurrent chemotherapy consisted of TMZ (supplied by Merck company) at a dose of $75 \mathrm{mg} / \mathrm{per} / \mathrm{m}^{2}$, given 7 days per week from the first day of radiotherapy until the last day of radiotherapy, but for no longer than 49 days. Patient should be seen weekly with complete blood picture (CBC), with general, neurological assessment, and management of TMZ dosing according to Table 1.

After a 4-week break, patients were then to receive 4 weekly adjuvant temozolomide at a dose of $150 / \mathrm{m}^{2} /$ day for first adjuvant cycle, can be escalated to $200 \mathrm{mg} / \mathrm{m}^{2} /$ day during next cycles so long as there were no hematologic toxic effects. If ANC was $<1.0 \times 10^{9} / \mathrm{L}$, platelet count $<50 \times 10^{9} / \mathrm{L}$, and if CTC non hematological toxicity (except for alopecia, nausea, vomiting) is CTC Grade 3, this may mandate reducing the dose to $100 \mathrm{mg} / \mathrm{m}^{2} /$ day. If toxicity mandates further reduction TMZ should be stopped (Table 2).

Anti Pneumocystis pneumonia (PCP) prophylactic Trimethoprim-sulfamethoxazole $960 \mathrm{mg}$ was offered every other day during CCRT [12]. Antiemetic was offered before the initial doses of concurrent TMZ and per demand during the adjuvant five-day courses of TMZ. Anti-epileptics were offered for patients who have history of seizures.

All patients should be assessed clinically and neurologically on presentation. All patients should undergo baseline contrasted neuro-imaging with magnetic resonance imaging (MRI) or CT scan of 2.5 to $3 \mathrm{~mm}$ slice thickness. MRI more preferred, where the T1 with contrast series can be used as fusion images with the CT simulation planning for better gross tumor volume (GTV) delineation. CBC, renal and liver functions tests to be done one week before chemotherapy.

The planning CT scan is extended to encompass the head and neck region (At least up to C4 levels) to allow sufficient anatomic areas for proper image fusion and to permit the introduction of non-coplanar beams

Patients were seen weekly during CCRT, and 3 - 4 weeks after the completion of radiotherapy. During adjuvant temozolomide therapy, Patients were evaluated for response using clinical and neurological examinations performed monthly before each cycle and MRI or CT neuro-imaging performed after 6 months of adjuvant TMZ and then 3 monthly afterword. Imaging can be done before 6 months if clinically indicated. MRI spectroscopy or CT/PET scan (Positron Emission Tomography) can be done for better differentiation of progression versus post treatment effects (pseudo-progression). Patients who finished 6 cycles of adjuvant TMZ without clinical and radiological evidence of disease progression were randomized either to follow up or to continue TMZ until disease progression.

Responses were confirmed as complete or partial if they were constant at subsequent scans obtained at least 4 weeks apart from each other. Progression defined as increase in tumor size by 20 percent, the appearance of new lesions, or an increased need for corticosteroids [13] (see Appendix).

Table 1. TMZ dose modification for CCRT.

\begin{tabular}{cccc}
\hline Toxicity & Interrupt & Resume & Stop \\
\hline ANC $\left(\times 10^{9} / \mathrm{L}\right)$ & $\geq 0.5-<1.5$ & $\geq 1.5$ & $<0.5$ \\
Platelet $\left(\times 10^{9} / \mathrm{L}\right)$ & $\geq 10-<100$ & $\geq 100$ & $<10$ \\
NH toxicity & $\mathrm{G} 2$ & $\leq \mathrm{G} 1$ & $\mathrm{G} 3 / 4$ \\
\hline
\end{tabular}

ANC: Absolute Neutrophil Count, Plat: Platelet count, NH toxicity: non hematological toxicity (except for alopecia, nausea, vomiting) CTC.2: Common Toxicity Criteria 2.

Table 2. Dose modification of adjuvant TMZ.

\begin{tabular}{ccccc}
\hline Dose level & Dose $\mathrm{mg} / \mathrm{m}^{2} / \mathrm{d}$ & Comments & Reduce TMZ by 1 dose level if: & Stop TMZ \\
\hline-1 & 100 & Reduction for prior toxicity & ANC $<1\left(\times 10^{9} / \mathrm{L}\right)$ & $\begin{array}{c}\text { if dose }<100 \mathrm{mg} / \mathrm{m}^{2} \text { is required } \\
\text { or if the same Grade } 3 \mathrm{NH} \\
\text { toxicity recurred after dose } \\
\text { reduction or became G4 }\end{array}$ \\
\hline
\end{tabular}

ANC: Absolute Neutrophil Count, Plat: Platelet count, NH toxicity: non hematological toxicity (except for alopecia, nausea, vomiting) CTC.2: Common Toxicity Criteria 2. 
Neurological status was assessed by considering signs and symptoms possibly correlated with progression, as compared to the previous examination; each variation in daily corticosteroids dosage was recorded. When there was tumor progression or patients were treated at the investigator's discretion, and the type of second-line therapy was recorded.

Progression free survival (PFS) was calculated from the date of initial surgery to progression on MR imaging, or the date of last contact or death. Overall survival (OS) was calculated from the date of diagnosis to the date of last contact or death. Toxic effects were graded according to the National Cancer Institute Common Toxicity Criteria, version 2.0.

Statistics was done through IBM SPSS statistics v20.0 program. Median PFS and median overall survival were calculated using the Kaplan-Meier method (Kaplan \& Meier, 1958), and hazard ratio with Cox-regression model; differences in progression and overall survival (OS) were evaluated by the log-rank test for statistical significance.

\section{Results}

59 patients were recruited to the study. The median age was 55 years, range (19 - 72), among them 47 (79.7\%) were males and 12 (20.1\%) were females. The sex ratio was 3.9. Median KPS at diagnosis was 80 (60 - 100) and the median of NPS was $1(0-3)$. Histopathological diagnosis showed GBM in the entire patient, 5 patients had multiple tumors (8.4\%). Patient characteristics before treatment are summarized in Table 3.

Patients were randomized after diagnosis by intension-to-treat (ITT) into two groups. Twenty nine patients (49.2\%) were randomized to receive 6 cycles of adjuvant TMZ after the end of CCRT and 30 patients (50.8\%) to receive more than 6 cycles after the end CCRT ( $>6$ cycles arm). The patients were well randomized regarding gender, age, KPS and NPS. See Table 4.

\section{Table 3. Overall patient characteristics.}

\begin{tabular}{|c|c|}
\hline Character & No (\%) \\
\hline Overall patient & $59(100 \%)$ \\
\hline 6 cycles arm & $29(49.2 \%)$ \\
\hline$>6$ cycles arm & $30(50.8 \%)$ \\
\hline \multicolumn{2}{|l|}{ Gender } \\
\hline Males & $47(79.7 \%)$ \\
\hline Females & $12(20.1 \%)$ \\
\hline \multicolumn{2}{|l|}{ KFS performance status } \\
\hline Median & 80 \\
\hline Range & $(60-100)$ \\
\hline \multicolumn{2}{|l|}{ NPS } \\
\hline Median & 1 \\
\hline Range & $(0-3)$ \\
\hline \multicolumn{2}{|l|}{ Multiplicity } \\
\hline Solitary & 54 (91.5\%) \\
\hline Multiple & $5(8.4 \%)$ \\
\hline \multicolumn{2}{|l|}{ Surgical intervention } \\
\hline Biopsy only & $11(18.6 \%)$ \\
\hline Subtotal resection & $21(35.6 \%)$ \\
\hline Total resection & $27(45.8 \%)$ \\
\hline
\end{tabular}


Table 4. Criteria of both treatment arms.

\begin{tabular}{|c|c|c|c|}
\hline Characteristic & 6 cycles arm $n=29(49.1 \%)$ & $>6$ cycles arm $n=30(50.8 \%)$ & $P$ value \\
\hline Gender & & & (P 0.5) \\
\hline Male & $20(68 \%)$ & $23(67.7 \%)$ & \\
\hline Female & $9(31 \%)$ & $7(23.3 \%)$ & \\
\hline Age (mean \pm SD) & $53.0 \pm 10.9$ & $55.9 \pm 8.6$ & (P 0.51) \\
\hline KPS (median (range)) & $80(60-100)$ & $80(60-100)$ & (P 0.93) \\
\hline NFS (median (range)) & $1(0-3)$ & $1(0-3)$ & (Р 0.26) \\
\hline
\end{tabular}

Among the whole group of patients 11 (18.6\%) patients underwent only biopsy, 7 of those biopsies was planned because of deep seated tumors, or multiplicity. Forty eight patients (81.3\%) had maximal safe resection. Twenty one (35.6\%) had subtotal resection and 27 (45.8\%) had a gross total resection. Only 53 (89.8\%) received postoperative CCRT, while 6 patient had only surgeries. The mean total dose of RT was (59 Gy \pm 2.4 SD) (range 54 - 62). Thus overall 53 patient started the CCRT, $26(89.6 \%)$ at 6 cycles arm, and 27 (90\%) at >6 cycles arm. Among those 53 patients who started the CCRT, only 43 (81.1\%) finished the planned full dose chemoradiotherapy and 10 (18.8\%) patients did not complete it, 5 patients in each arm. Three out of those 10 patients stopped the CCRT because of hematological toxicity. Thus 43 patients started to receive the adjuvant TMZ, 21 (72.4\%) patients of the 6 cycles arm and 22 (73.3\%) patients among the $>6$ cycles arm.

Out of the 21 patient in the 6 cycles arm, 16 patients finished the planned 6 cycles of adjuvant TMZ, while 5 (17.2\%) patients received less than 6 cycles. Two patients received 3 cycles, one patient received 4 cycles and another 2 received 5 cycles only. Among the $>6$ cycles arm 3 patients (13.6\%) received also less than 6 cycles, 2 received 2 cycles and one received 4 cycles. While 19 patients $(86.3 \%)$ received more than 6 cycles. The median number of extended cycles of adjuvant TMZ for those patients was 11 cycles, and range (8 - 23). See Table 5.

The patients were followed up for median of 15.2 months (range 2 - 49.1 months). 58 patients died. See Table 6. The Kaplan-Maier analysis showed median progression free survival (PFS) for all patients with ITT in the 6 cycles arm is 10.4 month [CI 95\% 8.1 - 12.6] and for >6 cycles arm 13.2 months [CI 95\% 8.5 - 17.8] (P 0.038) (Figure 1(a)). Overall survival for whole 6 cycles arm versus $>6$ cycles arm are 14.1 months [95\% CI 10.4 17.7] versus 18.8 months [95\% CI 11.9 - 25.6] (P 0.07) (Figure 1(b)).

While for the patient in the 6 cycles arms that completed 6 cycles of adjuvant TMZ the PFS was 12.1 months [95\% CI 9.3 - 14.8) while for those in the >6 cycles arm who received prolonged doses was 18.8 months [95\% CI 14.8 - 22.7]. Cox-regression model analysis showed HR 0.88 [95\% CI 1.185 - 4.901] (P 0.015) (Figure 2(a) and Figure 2(b)).

Univariate analysis (ANOVA) showed statistical for $>6$ cycles of adjuvant TMZ (P 0.026), KFS performance status (P 0.46), and NPS (P 0.00), and not significant for age (P 0.63) or gender (P 0.73). However in Multivariate analysis (MANOVA) it showed only significance of $>6$ cycles (P 0.036).

Regarding toxicity, almost all G3-4 toxicity only encountered during CCRT.

Four (7.5\%) patients out of 53 started the CCRT their weekly CBC showed persistent G3/4 hematological toxicity (3 thrombocytopenia and 1 neutropenia), which imposed stoppage of concurrent TMZ.

Adjuvant TMZ was well tolerated. Overall 5 (11.6\%) patients reported thrombocytopenia, neutropenia. And 2 (4.6\%) patients reported both. One patient had anemia (2.3\%). Most of those hematological toxicity with adjuvant treatment was transient and without significant clinical implication other than short treatment delay and dose modification. Median time to relapse after the end of adjuvant chemotherapy in patients that completed CCRT only was 2.5 months, for those who finished at least 6 cycles of adjuvant TMZ is 4.1 months, and for those who received extended adjuvant TMZ is 6.8 months.

Overall 5 patients underwent re-surgery. All were in the patients who finished at least 6 cycles of adjuvant TMZ, 3 (18.8) in the 6 cycles arm and 2 (10.8) in the >6 cycles arm. Overall 20 (33.8\%) patient received TMZ upon disease progression. Out off those patients 6 (30\%) received the TMZ by alternative daily schedules. Eight patients out of the 59 patients (13.5\%) only received salvage bevacizumab on the first or second progression. Three patients were in the $>6$ cycles arm, and 4 patients in the 6 cycles arm. 
Table 5. Treatment disposition per arm.

\begin{tabular}{|c|c|c|}
\hline Procedure & 6 cycles arm $(n=29)$ & $>6$ cycles arm $(n=30)$ \\
\hline \multicolumn{3}{|l|}{ Surgery } \\
\hline Biopsy & $6(20.7 \%)$ & $5(16.7 \%)$ \\
\hline Partial resection & $9(31.0 \%)$ & $12(40.0 \%)$ \\
\hline Total gross resection & $14(48.3 \%)$ & $(43.3 \%)$ \\
\hline \multicolumn{3}{|l|}{ Treatment events } \\
\hline -Surgery only & $3(10.3 \%)$ & $3(10 \%)$ \\
\hline -Surgery CCRT & $26(89.6 \%)$ & 27 (90\%) \\
\hline -Radiotherapy to PTV (mean) & 59.0 Gy $\pm($ SD 2.58) & $58.97 \mathrm{~Gy} \pm(\mathrm{SD} 2.23)$ \\
\hline -Completed CCRT & $21(72.4 \%)$ & $22(73.3 \%)$ \\
\hline \multirow{2}{*}{ (Cause of un completion) } & $1(3.8 \%)$ & $2(7.4 \%)$ \\
\hline & $4(15.3 \%)$ & $3(11.1 \%)$ \\
\hline \multicolumn{3}{|l|}{-Adjuvant TMZ } \\
\hline$<6$ cycles adjuvant TMZ & $5(17.2 \%)$ & $3(13.6 \%)$ \\
\hline 6 cycles adjuvant TMZ & $16(76.1 \%)$ & \\
\hline$>6$ cycles adjuvant $T M Z$ & & 19 (86.3\%) \\
\hline Median No. of cycles & & 11 \\
\hline (Range) & & (8 - 23 cycle) \\
\hline Completed CCRT \& adjuvant & $\mathrm{N}=16(55.1 \%)$ & $\mathrm{N}=19(63.3 \%)$ \\
\hline Median PFS (month) & 12.1 & 18.8 (Р 0.01) \\
\hline Median OS (month) & 18.1 & 24.1 (P 0.04) \\
\hline Median time to progression from last TMZ dose (month) & $4.1(1.3-16.5)$ & $6.8(1.8-20)$ \\
\hline \multicolumn{3}{|l|}{ Treatment post progression } \\
\hline Surgery & $4(25.0 \%)$ & $5(26.3 \%)$ \\
\hline TMZ 4 weekly & $5(31.3 \%)$ & $3(31.6 \%)$ \\
\hline TMZ alternative schedule & $3(18.8 \%)$ & $2(10.5 \%)$ \\
\hline Bevacizumab & $3(18.8 \%)$ & $4(21.0 \%)$ \\
\hline All patients & $N=29$ & $\mathrm{~N}=30$ \\
\hline Median PFS (month) & 10.4 & 13.2 (Р 0.038) \\
\hline Median OS (month) & 14.1 & 18.8 (Р 0.07) \\
\hline \multicolumn{3}{|l|}{ Treatment post progression } \\
\hline Surgery & $4(13.7 \%)$ & $5(16.6 \%)$ \\
\hline TMZ 4 weekly & $6(20.7 \%)$ & $8(26.7 \%)$ \\
\hline TMZ alternative schedule & $3(10.3 \%)$ & $3(10.0 \%)$ \\
\hline Bevacizumab & $4(13.7 \%)$ & $4(13.3 \%)$ \\
\hline \multicolumn{3}{|l|}{ G3/4 toxicity } \\
\hline On CCRT & $N=26$ & $\mathrm{~N}=27$ \\
\hline Thrombocytopenia & $1(3.8 \%)$ & $2(7.4 \%)$ \\
\hline Neutropenia & $1(3.8 \%)$ & 0 \\
\hline On adjuvant $T M Z$ & $N=21$ & $\mathrm{~N}=22$ \\
\hline Thrombocytopenia & $1(4.7 \%)$ & $2(9.0 \%)$ \\
\hline Neutropenia & $2(7.6 \%)$ & $4(18 \%)$ \\
\hline Anemia & 0 & $1(4.5 \%)$ \\
\hline
\end{tabular}




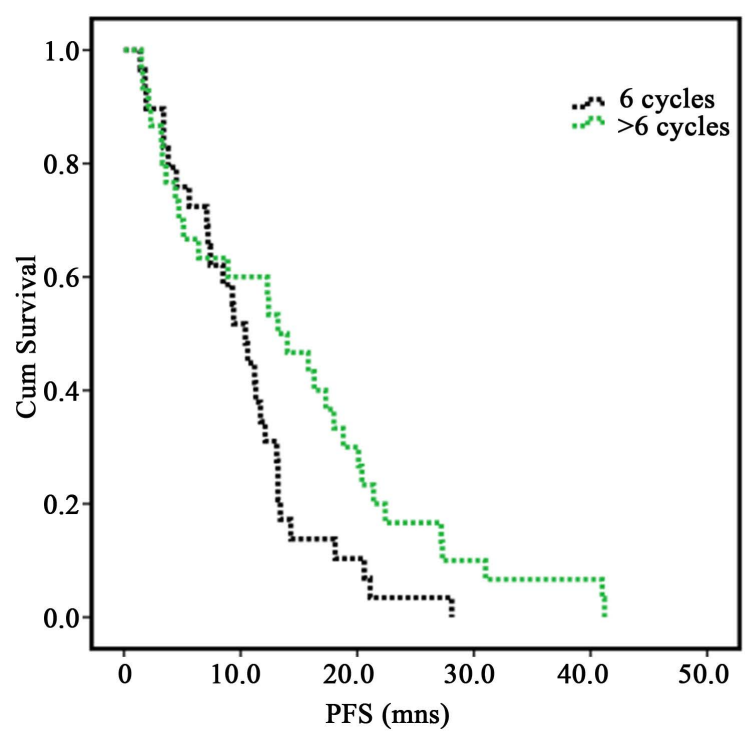

(a)

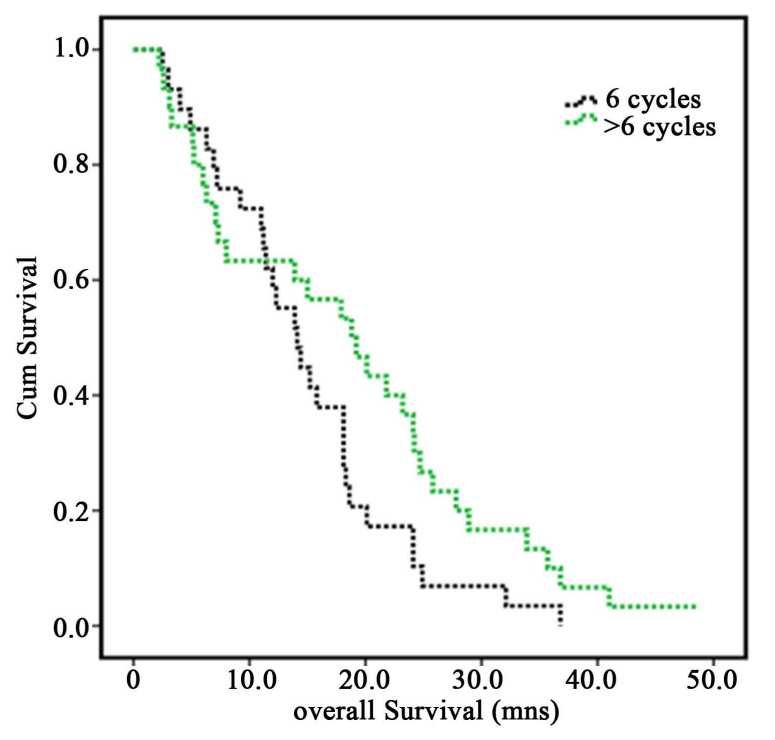

(b)

Figure 1. PFS and OS for whole 6 cycles arm and >6 cycles arms (ITT). (a) Median PFS 10.4 vs. 13.2 months (P 0.041); (b) Median OS 14.1 vs. 18.8 months (P 0.070).

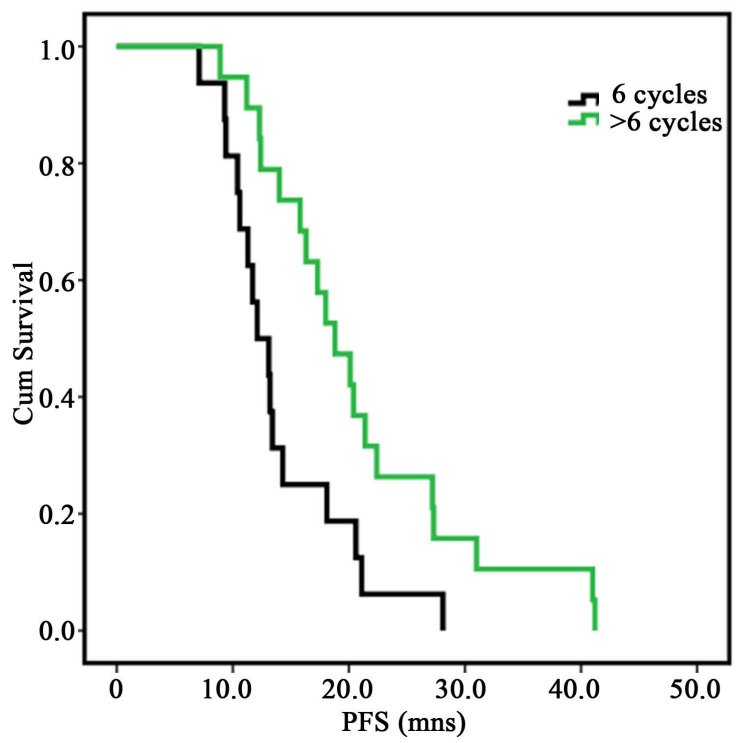

(a)

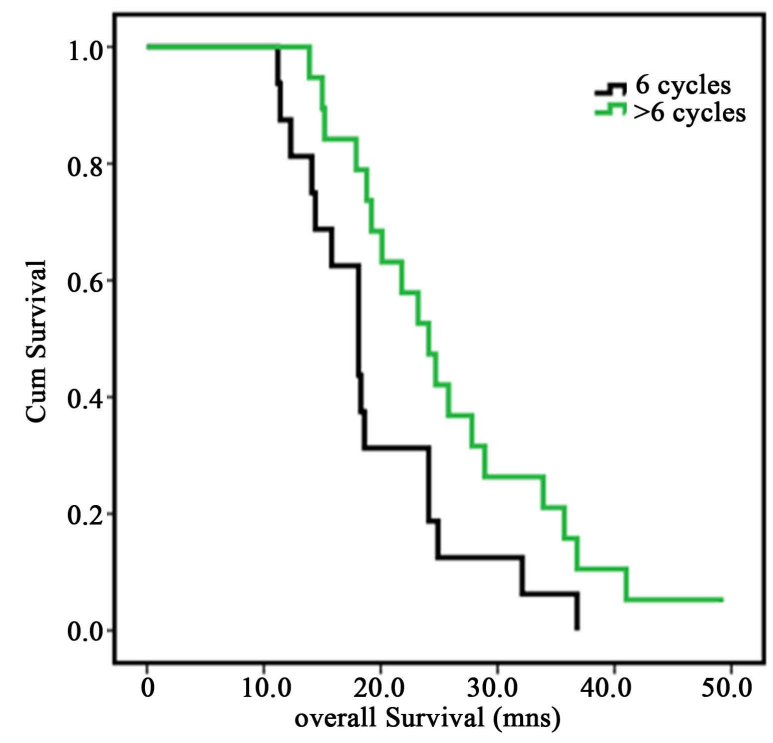

(b)

Figure 2. PFS and OS for 6 cycles arm (who completed whole 6 cycles) vs. $>6$ cycles that received extended adjuvant cycles. (a) Median PFS 12.1 vs. 18.8 months (P 0.015); (b) Median OS 18.1 vs. 24.1 months (P 0.048).

Table 6. Overall survival.

\begin{tabular}{|c|c|c|c|c|c|c|}
\hline Group & No. at risk & $12 \mathrm{mo}$ & $18 \mathrm{mo}$ & $24 \mathrm{mo}$ & $36 \mathrm{mo}$ & $48 \mathrm{mo}$ \\
\hline All patients & 59 & (37) $62.7 \%$ & (26) $44 \%$ & (16) $27.1 \%$ & (6) $10.1 \%$ & (1) $1.6 \%$ \\
\hline 6 cycles (ITT) & 29 & (18) $62.0 \%$ & (11) $37.9 \%$ & (05) $17.2 \%$ & (1) $3 \%$ & (0) $0.0 \%$ \\
\hline >6 cycles $(\mathrm{ITT})$ & 30 & (19) 63.3\% & (15) $50 \%$ & (11) $36.6 \%$ & (5) $16 \%$ & (1) $3.0 \%$ \\
\hline 6 cycles (completed) & 16 & (14) $87.5 \%$ & (10) $62.5 \%$ & (05) $31.2 \%$ & (1) $6.2 \%$ & (0) $0.0 \%$ \\
\hline$>6$ cycles (completed) & 19 & (19) $100 \%$ & (15) $78.9 \%$ & (10) $52.2 \%$ & (3) $15.7 \%$ & (1) $5.2 \%$ \\
\hline
\end{tabular}

ITT: intension to treat. 


\section{Discussion}

GBM is the most common of all primary brain tumors and is associated with a median survival of approximately 5 months without treatment [14]. Before the wide use of temozolomide, chemotherapy achieved only small survival benefit. A Meta analysis published on 2002 based on 12 randomized trials investigating the efficacy of chemotherapy in glioma, showed a 5\% increase in the 2-year survival (15\% vs. 20\%). However it included 37\% low grade glioma with definitely more favorable prognosis [15].

Stupp and colleagues study that used CCRT, and 6 cycles of adjuvant 6 cycles showed a median overall survival of 14.6 months vs. 12.1 months for radiation only. And the mature result on 2009 demonstrated a persistent survival advantage up to 5 years (1.9\% versus 9.8\%) proving long lasting efficacy of TMZ [4]. However $73.5 \%$ die within the first two years, and still we have long way to go to improve the outcome. With lack of an effective second line treatment, ideas went to extending the adjuvant TMZ doses, use daily metronormic doses or adding new agent.

Holdhoff and Stuart denied the extended adjuvant strategy based the built up experience with chemotherapy in other solid tumors suggested maximum tumor cell kill is achieved with the first two to four cycles of chemotherapy. With no chemotherapy till disease progression did not improve the overall survival [16].

In our study we recruited 59 patient randomized in two groups for either 6 cycles of adjuvant treatment or more than 6 cycles. Progression free survival and overall survival included those who managed to finish the treatment plan, and for the whole arm based on intension to treat analysis.

For arm one (6 cycles only arm) only 16 out of 29 patients completed the CCRT and 6 cycles of adjuvant TMZ (55.1\%) and 19 out of 30 (63.3\%) in arm two (more than 6 cycles) did the same with add extra doses of adjuvant TMZ.

Close results showed up in many trials. In the EORTC/NCIC study, 105 out of 287 patients (47\%) in the radiotherapy and chemotherapy arm finished 6 cycles of adjuvant TMZ [3].

In a study by Delion and Moraru on 273 glioma patients 115 patients (42\%) received adjuvant monthly cycles of TMZ, and out of them in this group 60 patients (52\%) had radio-logical evidence of disease progression before completing six cycles of adjuvant TMZ [17].

In a retrospective French study by Darlix et al. examined files of 448 patients, 201 patients received Stupp protocol and 78 (38.8\%) patients managed to finish it [18].

Radiological assessment was done late by the end of the first 6 adjuvant TMZ and not earlier to avoid the treatment induced necrosis. Pseudo-progression is a well known phenomenon, explained by injury to the blood brain barrier by both radiotherapy and chemotherapy and is seen in $20 \%-30 \%$ of cases may show up after 1 month of CCRT and last for many months [19]-[21] it is.

Brandes and Franceschi suggested that pseudo progression resulted in over estimation of disease progression in CCRT arm in EORTC/NCIC, and this is why a small improvement of PFS of 6.8 months vs. 5 months was translated to consistent survival benefit which is not common in oncology [22].

Unfortunately, other radiologic studies, such as positron emission tomography or nuclear magnetic resonance spectroscopy, have not been useful in this setting. As a result, in selected cases, tissue may be required to distinguish treatment-related effects from progressive neoplasm if clinically indicated.

In our study the median number of cycles in the (>6 cycles) group was 11, ranging from 8 - 23 cycles. This is similar to the study by Delion and Moraru in which the median was also 11 and range 7 - 13 [17]. While in the French study the median was 20 ranging 9 - 26, and actually they excluded patient with less than 9 cycles from being analyzed among the prolonged administration group. They anticipated that no expected major difference between 6 and less than 9 cycles [18]. In another study over 114 although the median adjuvant cycles number was 6 however some patient received up to 57 cycles [23]. Moreover Khasraw reported one patient received to 8 years of adjuvant TMZ [9].

The Median PFS for (>6 cycles) arm was 18.8 months vs. 12.1 months (P 0.01). The median overall survival for the ( $>6$ cycles) arm was 24.1 months vs. 18.1 months for the standard 6 cycles arm (P 0.04). The 2-year survival for the same groups were $52.2 \%$ vs. $31.2 \%$, and for the whole patient is 27.1 months.

These results are very similar to the French study with median survival for the 6 cycles arm of 16.5 months, and for the extended adjuvant arms of 24.6 months (P 0.031) [24].

If we compared these result with available data we find that while the all patients and the 6 months arm does not differ much from the 2 years survival in the EORTC/NCIC study with 2-year survival of 26.5 months and 
median OS of 14.6 month, however it apparently do better among those in (>6 cycles) arm who received TMZ till disease progression.

However this result is far less than the result showed by Darlix ET. Al. it showed PFS of 28.4 month (range 12.8 - 34.2), 2-year survival of $76 \%$, and 3 years survival of $48 \%$ for patients that received 9 cycles or more. However the prognosis in such result as a whole in both the standard 6 cycles and more was good, with $69.4 \%$ alive 24 months after diagnosis compared with $27.2 \%$ in the RT-TMZ arm of the EORTC-NCIC trial [18]. The authors attributed this impressive results $60.3 \%$ of complete tumor excision, and randomization was done among patients managed to finish the adjuvant 6 cycles of adjuvant TMZ.

Regarding toxicity, which is the other factor that can limit the prolonged administration of chemotherapy generally including TMZ, it was of limited effect overall. TMZ may cause myelosuppression and mainly thrombocytopenia, observed in approximately $12 \%$ - 20\% of patients [3] [25].

Also known that RT, TMZ, and dexamethasone have a profound effect on total lymphocyte and CD4 counts, thus it requires prolonged Pneumocystis jiroveci prophylaxis [26], which was routinely offered for all patients who started the CCRT.

In our patients, almost all G3-4 toxicity only encountered during CCRT. Out of 53 started the CCRT, 4 patients' encountered G3-4 hematological toxicity which imposed stoppage of concurrent TMZ, while during the adjuvant 4 weekly TMZ the hematological toxicity was transient and managed without significant impact on treatment.

Such finding is almost constant in most of the published data. Hau et al., series had few grade 3 - 4 toxicities [6]. Malkom et al., in their retrospective study stated 8out of 46 patients (17.3\%) required dose adaptation because of side effects in the adjuvant phase of their retrospective study [8], and 1patient out of 58 (1.7\%) had G4 lymphopenia grade during the additional cycles of TMZ (after the eighth), with no clinical consequence [18].

In univariate analysis, more than 6 cycles of adjuvant chemotherapy, KFS performance status and NPS showed improvement of survival, with no impact on of age or gender. While in multivariate analysis it is only the prolonged adjuvant that showed improvement of Survival (P 0.036). In the French study the multivariate analysis showed effect only to CCRT (P 0.01) and the extended adjuvant treatment (P 0.014) with no effect to age, KPS, debulking, or MGMT promoter [24].

Our study is prospective and showing results lumping with other retrospective studies and clinical practice advocate prolonged adjuvant TMZ till disease progression. However the number who managed to receive such treatment was small, and salvage treatment was not uniformal range from surgery to chemotherapy and bevacizumab.

\section{Conclusion}

Adjuvant TMZ post concurrent chemoradiotherapy till disease progression after in patients with GBM is feasible and well tolerated in most patients. Accumulated data, including this study, may suggest this strategy for improving outcome of GBM patients post 6 cycles with reasonable PS and still possible residual. A phase III study with large number of patient is required to support this conclusion.

\section{References}

[1] Wen, P.Y. and Kesari, S. (2008) Malignant Gliomas in Adults. New England Journal of Medicine, 359, 492-507. http://dx.doi.org/10.1056/NEJMra0708126

[2] Ricard, D., et al. (2012) Primary Brain Tumours in Adults. Lancet, 379, 1984-1996. http://dx.doi.org/10.1016/S0140-6736(11)61346-9

[3] Stupp, R., et al. (2005) Radiotherapy plus Concomitant and Adjuvant Temozolomide for Glioblastoma. New England Journal of Medicine, 352, 987-996. http://dx.doi.org/10.1056/NEJMoa043330

[4] Stupp, R., et al. (2009) Effects of Radiotherapy with Concomitant and Adjuvant Temozolomide versus Radiotherapy Alone on Survival in Glioblastoma in a Randomised Phase III Study: 5-Year Analysis of the EORTC-NCIC Trial. Lancet Oncology, 10, 459-466. http://dx.doi.org/10.1016/S1470-2045(09)70025-7

[5] Newlands, E.S., et al. (1997) Temozolomide: A Review of Its Discovery, Chemical Properties, Pre-Clinical Development and Clinical Trials. Cancer Treatment Reviews, 23, 35-61. http://dx.doi.org/10.1016/S0305-7372(97)90019-0

[6] Hau, P., et al. (2007) Safety and Feasibility of Long-Term Temozolomide Treatment in Patients with High-Grade Glioma. Neurology, 68, 688-690. http://dx.doi.org/10.1212/01.wnl.0000255937.27012.ee 
[7] Seiz, M., et al. (2010) Long-Term Adjuvant Administration of Temozolomide in Patients with Glioblastoma Multiforme: Experience of a Single Institution. Journal of Cancer Research and Clinical Oncology, 136, 1691-1695. http://dx.doi.org/10.1007/s00432-010-0827-6

[8] Malkoun, N., et al. (2012) Prolonged Temozolomide for Treatment of Glioblastoma: Preliminary Clinical Results and Prognostic Value of p53 Overexpression. Journal of Neuro-Oncology, 106, 127-133. http://dx.doi.org/10.1007/s11060-011-0643-0

[9] Khasraw, M., Bell, D. and Wheeler, H. (2009) Long-Term Use of Temozolomide: Could You Use Temozolomide Safely for Life in Gliomas? Journal of Clinical Neuroscience, 16, 854-855. http://dx.doi.org/10.1016/j.jocn.2008.09.005

[10] Mason, W.P., et al. (2007) Canadian Recommendations for the Treatment of Glioblastoma Multiforme. Current Oncology, 14, 110-117. http://dx.doi.org/10.3747/co.2007.119

[11] Bokstein, F., et al. (2008) A Common Sense Approach to Radiotherapy Planning of Glioblastoma Multiforme Situated in the Temporal Lobe. International Journal of Radiation Oncology Biology Physics, 72, 900-904. http://dx.doi.org/10.1016/j.ijrobp.2008.01.053

[12] Kovacs, J.A. and Masur, H. (2000) Prophylaxis against Opportunistic Infections in Patients with Human Immunodeficiency Virus Infection. The New England Journal of Medicine, 342, 1416-1429. http://dx.doi.org/10.1056/NEJM200005113421907

[13] Macdonald, D.R., et al. (1990) Response Criteria for Phase II Studies of Supratentorial Malignant Glioma. Journal of Clinical Oncology, 8, 1277-1280.

[14] Ohgaki, H. and Kleihues, P. (2005) Population-Based Studies on Incidence, Survival Rates, and Genetic Alterations in Astrocytic and Oligodendroglial Gliomas. Journal of Neuropathology \& Experimental Neurology, 64, 479-489.

[15] Stewart, L.A. (2002) Chemotherapy in Adult High-Grade Glioma: A Systematic Review and Meta-Analysis of Individual Patient Data from 12 Randomised Trials. The Lancet, 359, 1011-1018. http://dx.doi.org/10.1016/S0140-6736(02)08091-1

[16] Holdhoff, M. and Grossman, S.A. (2011) Controversies in the Adjuvant Therapy of High-Grade Gliomas. Oncologist, 16, 351-358. http://dx.doi.org/10.1634/theoncologist.2010-0335

[17] Delion, M., et al. (2010) Glioblastoma Incident Studies from May 2006 to May 2007 in Angers and Nice, France. Neurochirurgie, 56, 499-502. http://dx.doi.org/10.1016/j.neuchi.2010.07.006

[18] Darlix, A., et al. (2013) Prolonged Administration of Adjuvant Temozolomide Improves Survival in Adult Patients with Glioblastoma. Anticancer Research, 33, 3467-3474.

[19] Kumar, A.J., et al. (2000) Malignant Gliomas: MR Imaging Spectrum of Radiation Therapy- and Chemotherapy-Induced Necrosis of the Brain after Treatment. Radiology, 217, 377-384. http://dx.doi.org/10.1148/radiology.217.2.r00nv36377

[20] Brandes, A.A., et al. (1998) Carboplatin and Teniposide Concurrent with Radiotherapy in Patients with Glioblastoma Multiforme: A Phase II Study. Cancer, 82, 355-361. http://dx.doi.org/10.1002/(SICI)1097-0142(19980115)82:2<362::AID-CNCR17>3.0.CO;2-X

[21] DeAngelis, L.M., Delattre, J.Y. and Posner, J.B. (1989) Radiation-Induced Dementia in Patients Cured of Brain Metastases. Neurology, 39, 789-796. http://dx.doi.org/10.1212/WNL.39.6.789

[22] Brandes, A.A., et al. (2008) MGMT Promoter Methylation Status Can Predict the Incidence and Outcome of Pseudoprogression after Concomitant Radiochemotherapy in Newly Diagnosed Glioblastoma Patients. Journal of Clinical Oncology, 26, 2192-2197. http://dx.doi.org/10.1200/JCO.2007.14.8163

[23] Seiz, M., et al. (2010) Long-Term Adjuvant Administration of Temozolomide in Patients with Glioblastoma Multiforme: Experience of a Single Institution. Journal of Cancer Research and Clinical Oncology, 136, 1691-1695. http://dx.doi.org/10.1007/s00432-010-0827-6

[24] Roldan Urgoiti, G.B., Singh, A.D. and Easaw, J.C. (2012) Extended Adjuvant Temozolomide for Treatment of Newly Diagnosed Glioblastoma Multiforme. Journal of Neuro-Oncology, 108, 173-177. http://dx.doi.org/10.1007/s11060-012-0826-3

[25] Gerber, D.E., et al. (2007) The Impact of Thrombocytopenia from Temozolomide and Radiation in Newly Diagnosed Adults with High-Grade Gliomas. Neuro-Oncology, 9, 47-52. http://dx.doi.org/10.1215/15228517-2006-024

[26] Grossman, S.A., et al. (2011) Immunosuppression in Patients with High-Grade Gliomas Treated with Radiation and Temozolomide. Clinical Cancer Research, 17, 5473-5480. http://dx.doi.org/10.1158/1078-0432.CCR-11-0774 


\section{Appendix}

\section{Neurologic Performance Scale (MRC)}

$0 \quad$ No neurological deficit.

1 Some neurological deficit, but function adequate for.

2 Neurological deficit causing moderate functional impairment, e.g. ability to move limps only with difficulty, moderate dysphasia, moderate paresis, some visual disturbances.

3 Neurological deficit causing major functional impairment, e.g.: inability to use limb/s, gross speech or visual disturbances. No useful function-inability to make conscious responses.

\section{Mcdonaled criteria for assessment of progression in glioma}

Complete Requires all of the following: complete disappearance of all enhancing measurable and non measurable response disease sustained for at least 4 weeks; no new lesions; no corticosteroids; and stable or improved clinically

Partial

response

Requires all of the following: $50 \%$ decrease compared with baseline in the sum of) products of perpendicular diameters of all measurable enhancing lesions sustained for at least 4 weeks; no new lesions; stable or reduced corticosteroid dose; and stable or Improved clinically

Stable

disease

Requires all of the following: does not qualify for complete response, partial response, or progression; and stable clinically

Progression

Defined by any of the following: $25 \%$ increase in sum of the products of perpendicular diameters of enhancing lesions; any new lesion; or clinical deterioration 